\title{
A Method for the Quantification of Total Xenon Concentration in Each Phase of MOX Irradiated Nuclear Fuel with SIMS and EPMA
}

\author{
K. Hanifi, L. Noirot, Ph. Bienvenu, J. Lamontagne, I. Aubrun-Zacharie, C. Pozo, J. Noirot, I. Roure, \\ Th. Blay, P. Delion, E. Brochard
}

\section{CEA-Cadarache, Nuclear Energy Division, Department of Fuel Studies, F-13108 St Paul Lez Durance France}

The behaviour of gases produced by fission is of great importance for nuclear fuel in operation. An experimental method for the quantitative determination of the total Xe concentration, including the gas trapped in bubbles, in each phase of an irradiated MOX nuclear fuel was developed in our laboratory.

MIMAS MOX fuels $\left(\mathrm{UO}_{2}-\mathrm{PuO}_{2}\right)$ are constituted of three phases with three Pu concentration levels called $\mathrm{Pu}$-poor agglomerates, intermediate phase, $\mathrm{Pu}$-rich agglomerates (Figure 1). Under irradiation, the behavior of each phase differs depending on the Pu content and on its radial position in the pellet. Indeed, the local burn-up in Pu-rich agglomerates is clearly higher than the pellets average burn-up. At the periphery of the pellets, the High Burn-up Structure (HBS) forms in these Pu-rich agglomerates for relatively low average fuel burn-ups [1]. In the central part of the pellets, where the irradiation temperatures are higher, the Pu-rich agglomerates exhibit also major changes, with the formation of a very large pore and large metallic fission product precipitate formation.

Given these differences in microstructure and the complexity of the MOX fuel, methods had to be developed to measure the local Xe distribution and its local production. For that, shielded SEM, EPMA, SIMS and confocal microscope were used for polished sections of irradiated MOX fuel rod examinations [2].

EPMA analyses involve only a layer at the sample surface, thinner than $1 \mu \mathrm{m}$, this thickness depending on the acceleration voltage of the electron beam. Within this analysed layer, part of the gas initially stored in bubbles escapes when the polished surface cuts these bubbles. The amount of this lost gas is not negligible for the measurement when the bubbles are larger than $\sim 0.02 \mu \mathrm{m}$ [3]. Consequently, the EPMA measurement of Xe in the fuel cannot provide a complete information on the Xe distribution. This is particularly true when applied to HBS zones where bubbles formed are often larger than $0.4 \mu \mathrm{m}$.

SIMS usually provides a local isotopic analysis of a solid sample, as illustrated in Figure 2. A specific method has been developed for the measurement of fission gases [4-6]. This method has been widely applied to irradiated $\mathrm{UO}_{2}$ fuels [7-11] and to irradiated MOX fuels [12].

On irradiated samples, containing a wide range of fission product isotopes, the Xe signal measured with SIMS must be separated from the molecular interferences produced by fission products, for example ${ }^{132} \mathrm{Xe}^{+}$with ${ }^{100} \mathrm{Mo}^{16} \mathrm{O}_{2}^{+}$. The $\mathrm{Xe}$ signal is too low to enable a separation with a high mass resolution tuning. So the interferences were avoided thanks to an energy filter: the ion energy depends on the location at which the atoms or molecules are ionized. The Xe ions are created over the sample surface while most of the other ions are created at the surface. This separation is illustrated in Figure 3.

The Xe filling the bubbles is detected as peaks on the Xe signal measured during the sputtering, while the Xe in solid solution or coming from the opening of nano bubbles form the base line of the depth profile [7]. Confocal microscope measurements give information on the volume of the craters, i.e. the amount of fuel sputtered during Xe measurement.

For a heterogeneous microstructure examination, such as MOX fuels, knowing the local production of Xe is almost as important as knowing the local content and distribution of this Xe. This is obtained through the local measurements of ${ }^{145} \mathrm{Nd}$ and ${ }^{146} \mathrm{Nd}$ isotopes, in each of the craters formed for the 
Xe measurement. Indeed, $\mathrm{Nd}$ is a fission product that forms a solid solution in $\mathrm{UO}_{2}$ and $(\mathrm{U}, \mathrm{Pu}) \mathrm{O}_{2}$ and does not show any sign of migration after its production. These two isotopes can be used to have an estimation of the local burn-up, hence of the local Xe production.

Moreover, for each crater, the local isotopic content in $\mathrm{Pu}$ and $\mathrm{U}$, allows identifying the local phase (Pu-poor agglomerate, intermediate phase or $\mathrm{Pu}$-rich agglomerate). It also allows the detection of cases where the craters, aimed to analyse one specific phase, involved in fact also one of the surrounding phase. This checking work can be complemented post SIMS by a SEM examination and EPMA Pu maps, so that the phases involved in the measurement can be known precisely.

In addition, detailed lines ( $<1 \mu \mathrm{m}$ spots, $2 \mu \mathrm{m}$ steps) of isotopic analyzes $(\mathrm{Nd}, \mathrm{Pu}, \mathrm{U} . .$.$) through all$ three phases can be performed. This refines the knowledge of each phase composition as a function of the radial position.

Furthermore, the PRODHEL model, used in the fuel behavior code ALCYONE and developed to calculate the helium production in the fuels [13], has been expanded to allow fuel composition calculation in each phase of the MOX fuels. Isotopic compositions calculated with PRODHEL have been compared with SIMS measurements in each phase. That showed a good consistency.

It is concluded that the microanalysis techniques SIMS, EPMA and SEM, used in association, prove to be very powerful tools for the characterization of the fission gases in irradiated nuclear MOX fuel and also validate fuel modeling.

\begin{tabular}{|c|c|c|}
\hline 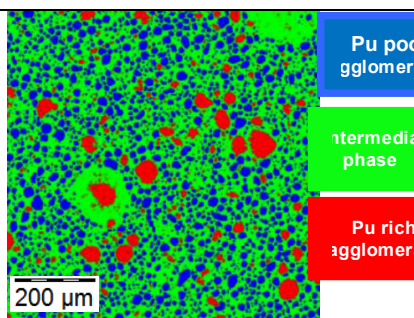 & 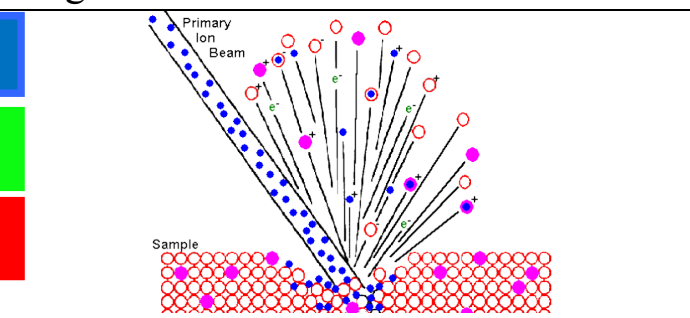 & 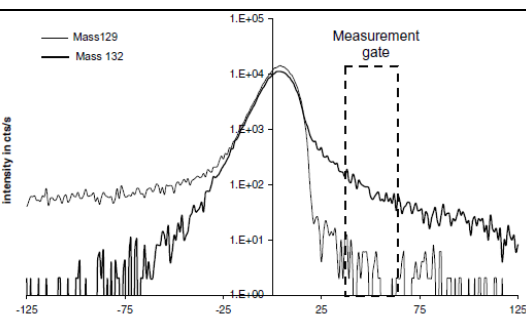 \\
\hline $\begin{array}{l}\text { Figure 1. The } 3 \mathrm{MOX} \\
\text { Fuel phases }\end{array}$ & $\begin{array}{l}\text { Figure 2. Schematic drawing of the } \\
\text { sample sputtering during SIMS analysis }\end{array}$ & $\begin{array}{l}\text { Figure 3. Energy spectra of } \\
129 \text { and } 132 \text { masses }\end{array}$ \\
\hline
\end{tabular}

\section{References:}

[1] J. Noirot, et al. J. Nucl. Mater. 372 (2008), p. 318.

[2] B. Rasser, et al Appl. Surf. Sci. 203-204 (2003), p. 673.

[3] M. Verwerft, J. Nucl. Mater. 282 (2000), p. 97.

[4] L. Desgranges, et al. NIM B 266 (2008), p. 147.

[5] J. Lamontagne et al, Microchim. Acta 145 (2004), p. 91.

[6] J. Noirot et al, Proc. Internat. Meeting on LWR Fuel Perf, Orlando, 2004.

[7] J. Lamontagne et al, Microchim. Acta 155 (2006), p. 183.

[8] J. Noirot et al. NET. 41 (2009), p. 155.

[9] J. Noirot et al. J. of Nucl. Mater. 442 (2013), p. 309.

[10] J. Noirot et al. J. Nucl. Mater. 446 (2014), p. 163.

[11] B. Marchand et al. J. Nucl. Mater. 440 (2013), p. 562.

[12] N. Nakae, et al. Top Fuel proceedings, Manchester, (2012).

[13] E. Federici, Proc. Internat. Meeting on LWR Fuel Perf., San Francisco, 2007. 